\title{
The Effect of Pressure on Electronic and Magnetic Properties of MnAs Crystal
}

\author{
Farzad Moradiannejad, S. Javad Hashemifar, and Hadi Akbarzadeh \\ Physics Department, Isfahan University of Technology, Isfahan 8415683111, Iran \\ Correspondence should be addressed to Farzad Moradiannejad; moradiannejad@alumni.iut.ac.ir
}

Received 18 June 2013; Revised 8 August 2013; Accepted 13 August 2013

Academic Editor: James Lutsko

Copyright ( 2013 Farzad Moradiannejad et al. This is an open access article distributed under the Creative Commons Attribution License, which permits unrestricted use, distribution, and reproduction in any medium, provided the original work is properly cited.

The structural, electronic, and magnetic properties of MnAs crystal are studied. The WIEN2k code which uses a full-potential LAPW program based on density functional theory with GGA is used for the calculations. At first, the total energy of a MnAs crystal in different lattices is calculated and the corresponding $E-V$ diagram is drawn for two different structures of MnAs. The effect of pressuring this crystal is determined. The calculations confirm that, MnAs has the NiAs-type structure at ambient pressure but transforms into the zinc-blend structure of a specific pressure value. Also, the electric field gradient (EFG) and hyperfine field (HFF) at the nuclear site of Mn and As are calculated. Finally, the effect of pressure on EFG and HFF is studied.

\section{Introduction}

Because of its structural, electronic, and magnetic properties, MnAs is a suitable compound for spin-electronic and magnetooptic applications. Recently its different properties were widely studied by the use of different experimental and computational methods [1-5]. Among the most important electronic properties of MnAs are its EFG and HFF which can be used to study the nonsymmetric part of the electron density around the nucleus and the core magnetic moment of the crystal. The effect of pressure on both EFG and HFF is studied which is useful in nuclear physics too. Nowadays, it is possible to study these two properties via computer simulation methods. In this paper, the effect of pressure on MnAs (in different crystal structures) is studied by density functional theory (DFT) calculations with the main emphasis on the EFG. The dependence of the EFG on pressure is related to change in electron charge density distribution [6].

The EFG is defined as the second derivative of the electrostatic potential at the nucleus site which is written as a traceless tensor $\left(V_{z z}\right)$. This tensor can be obtained from an integral over the nonspherical charge density. The coupling of an EFG to the quadrupole moment $(Q)$ of a nucleus causes a splitting of the nuclear energy levels which can be detected by the use of nuclear quadrupole resonance (NQR) measurements. The quadrupole resonance frequency $\left(v_{Q}\right)$ is obtained from the NQR measurement. If $Q$ and $v_{Q}$ are known, Then the EFG can be calculated [6-8]:

$$
H_{\mathrm{Q}}=\frac{1}{2} \sum_{i j} Q_{i j} V_{i j}, \quad v_{\mathrm{Q}}=\frac{e \mathrm{Q} V_{z z}}{h} .
$$

The nuclear quadrupole moments $(Q)$ can be measured in the laboratory (which is very important parameter in nuclear physics) for determining the charge distribution at the nucleus. But this practice is expensive and it has not enough precision. Its measurement error can be about $25 \%$ and it is not applicable for some nuclei [8]. When the EFG is calculated through an exact calculation method, $Q$ can be obtained more accurately than in the laboratory. The research studies show that, by calculations of the EFG at different pressures, the $Q$ measurement error can be decreased.

Hyperfine interaction techniques, based on the observation of the coupling between the nuclear moment and the internal field, have been widely used to study magnetic properties on an atomic scale. The total hyperfine field (HFF) can be decomposed into three terms such as a dominant Fermi contact term, a dipolar term, and an orbital contribution. Here, only the contact term, which in the scalar-relativistic 
limit is derived from the spin densities at the nucleus site, is calculated:

$$
H_{c}=\frac{8}{3} \pi \mu_{B}^{2}\left[\rho_{\uparrow}(0)-\rho_{\downarrow}(0)\right] .
$$

In the fully relativistic case, this spin density at the nucleus is replaced by its average over the Thomson radius $r_{T}=$ $Z e^{2} / m c^{2}$ [9].

For all calculations in this study, the full-potential linearized augmented plane wave (FP-LAPW) method [10] is used as implemented in the Vienna package WIEN2k [11]. It employs density functional theory with the generalized gradient approximation (GGA) for calculating the crystal properties.

For the study of pressure effects on the electronic and magnetic properties in crystals which have different structures, first each structure is studied in a pressure range (because pressure can cause a phase transition). The MnAs crystal has observed three different structures, the NiAstype structure as natural, the MnP-type structure in an experimental mode, and the zinc-blend mode which has not yet been seen in nature [12].

The properties of the NiAs and MnP types were experimentally studied, but the properties of the zinc-blend structure are theoretically predicted. One of the predictions is the lattice constant for the latter. For estimating the lattice constant, the $\mathrm{Ga}_{1-x} \mathrm{Mn}_{x}$ As compound is used which has the zinc-blend structure. In the laboratory, this lattice constant was measured only for some $x \ll 1$. By using a linear extrapolation to $x=1$, the lattice constant for MnAs with the zinc-blend structure has been calculated theoretically [12-14].

\section{Experimental and Computational Details}

The MnAs crystal has three different structures [12]. The first structure is the NiAs type with space group $P 63 / m m c$. In this structure, MnAs is a ferromagnetic metal. The experimental lattice constants are $a=3.722 \AA$ and $c=5.702 \AA[2,15]$.

The second structure is the MnP type with space group Pnma. In this structure MnAs has mixed ferromagnetic and antiferromagnetic properties. The experimental lattice constants are $a=4.053 \AA, b=5.495 \AA$, and $c=2.946 \AA$ [16].

The third structure is the zinc-blend structre with space group Fd3m. In this structure, MnAs is a semiconductor. The extrapolated computational lattice constant is $a=5.9 \AA$ [17].

Valence and semicore states are $3 p^{6} 4 s^{2} 3 d^{5}$ for $\mathrm{Mn}$ and $3 d^{10} 4 p^{3}$ for As. In the irreducible wedge of the Brillouin zone (IBZ), $120 k$-points are used. In the WIEN2K code, $R M T * k \max$ (smallest muffin tin radius times largest plane wave) is equal to 8.0 and the muffin tin nuclei for Mn and As atoms are taken as large as possible. This maximum value is equal to 2.3 Bohr. The nonspherical potential contribution in the Hamiltonian matrix taken to the upper limit for $L$ is 4 .

The calculations are based on the properties of the NiAstype structure. Therefore, the consistency of this structure in the pressure range should be determined. Based on experiments, by increasing the pressure up to $0.4 \mathrm{GPa}$, the structure of MnAs is changed to MnP type [18]. Since this latter structure mode has a complex of ferromagnetic and

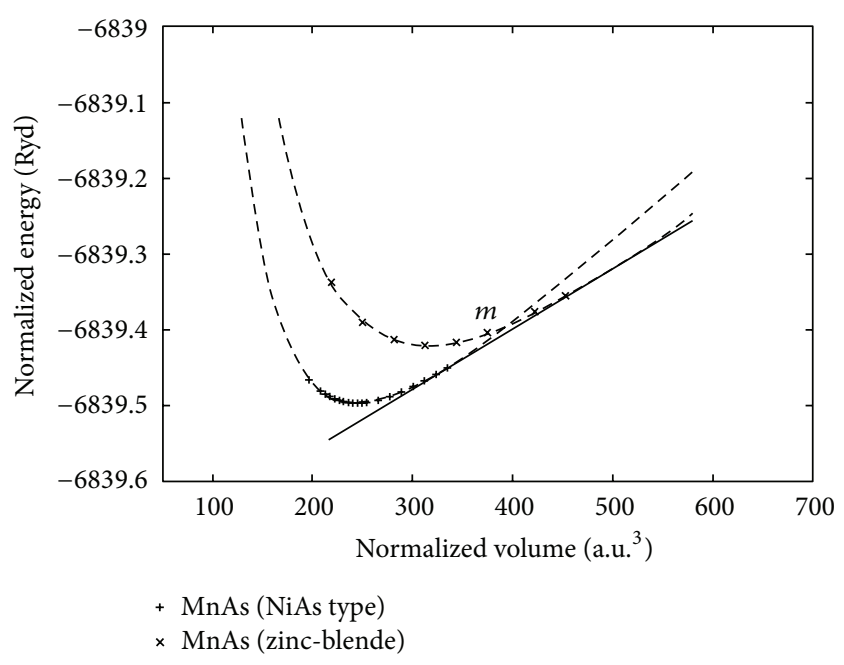

FIgURE 1: Energy-volume curve of MnAs with the NiAs and zincblend type.

antiferromagnetic properties, the structure simulation of this phase is very intricate. So, the experimental findings were used and $0.4 \mathrm{GPa}$ is taken as upper limit for pressure range.

In order to obtain the lower limit of pressure range, the $E$ $V$ curve is used. The derivative of the energy as a function of volume is equal to the unit of hydrostatic pressure on a crystal. Therefore, if the total energy as a function of volume for the two structures (NiAs-type and zinc-blend) is calculated and the pressure value of the phase transition is obtained from the common tangent between two $E-V$ curves (see Figure 1), then Gibbs energy is constant at the phase transition:

$$
G_{1}=G_{2} \longrightarrow E_{1}+P V_{1}=E_{2}+P V_{2} \Longrightarrow P=-\left.\frac{E_{2}-E_{1}}{V_{2}-V_{1}}\right|_{T=0} .
$$

Based on Figure 1, the lower limit of pressure is equal to $-0.126 \mathrm{GPa}$. Obtaining such a negative pressure value is impractical, but, for an enlarged lattice constant, it will be practical. For example it may be possible to grow an MnAs crystal over a substrate with a lager lattice constant such as a $\mathrm{Ga}_{1-x} \mathrm{Mn}_{x}$ As thin film [12]. This practice describes a negative pressure.

In Figure 1, the " $m$ " point shows that an MnAs crystal with a larger lattice constant than $5.86 \AA$ has a zinc-blend structure. This value agrees with other lattice constants calculated in preview references $[12,17]$. Therefore, the NiAs type has stable structure at all thermodynamically accessible pressures. Here, the electronic and magnetic properties of MnAs with the NiAs type structure are studied. In order to prevent a phase transition, the appropriate pressure range is $-0.126 \sim 0.4 \mathrm{GPa}$. The NiAs-type structure is shown in Figure 2. In this space group, there are two $\mathrm{Mn}$ atoms at $(1 / 3$, $2 / 3,1 / 4)$ and $(2 / 3,1 / 3,3 / 4)$ and two As atoms at $(0,0,0)$ and $(0,0,1 / 2)$. In the NiAs-type structure, the total energy and magnetization of MnAs are a function of the $c / a$ ratio [1]. In all calculations this ratio is assumed to remain constant. 


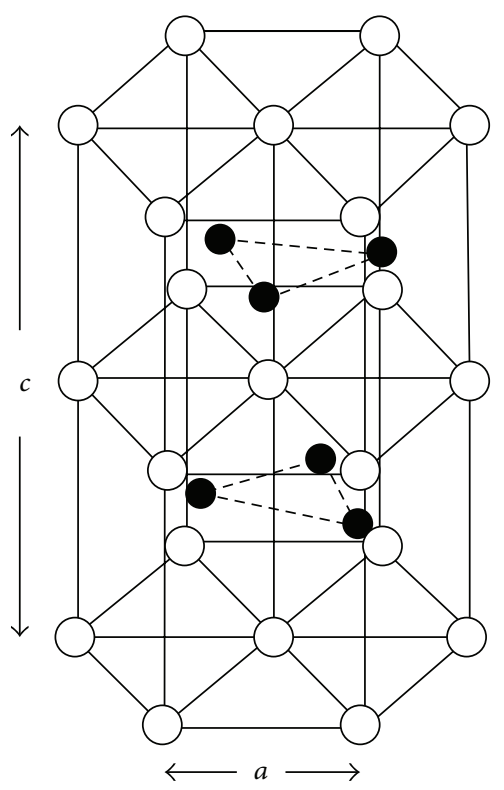

Figure 2: The conventional cell of MnAs with the NiAs-type structure.

\section{The Effect of Pressure on EFG}

Figure 3 shows the EFG values around the nucleus of the As ion at different pressures. At zero pressure, the EFG has a maximum value. By increasing (decreasing) pressure, the EFG decreases. In experiments, this behavior is observed for positive pressures. The decrease of the EFG value means that not only the distribution of electronic charge density around the nucleus but also the effects of other crystal ions (which is named EFG lattice portion) are changed by pressure such that the asymmetry of charge distribution decreases.

Figure 4 shows the EFG values around the nucleus of the $\mathrm{Mn}$ ion at different pressures. In this case, the maximum value of the EFG is observed in the structure with the experimental lattice constant. In both cases ( $\mathrm{Mn}$ and As), the EFG decreases with pressure. But at negative pressure, the EFG strongly depends on pressure. At $-0.0895 \mathrm{GPa}$, the EFG is zero. But beyond that pressure, a negative EFG would occur.

The asymmetry of electron charge density distribution around the nucleus is the main source for the EFG. Therefore, an analysis of the electronic charge density can be used to describe the behaviour of the EFG. Figure 5 shows the electron difference density contour plots which are taken from the difference between the electron density of the crystal and superposed atomic densities in the (030) plane. These contour plots are drawn for three different pressures $(0,-0.0895$, and -0.115$)$. In the contour plots, the negative density values are denoted by dashed lines. In Figure 4, the points of $\mathrm{A}, \mathrm{B}$ and $\mathrm{C}$ show the values of the EFG for $0,-0.0895$ and $-0.115 \mathrm{GPa}$. In Figure 5, the contour plots of these points are shown.

The sizes of contour-plot planes are selected such that only one $\mathrm{Mn}$ ion lies in the center of each plane. The asymmetry of the electron charge density distribution around

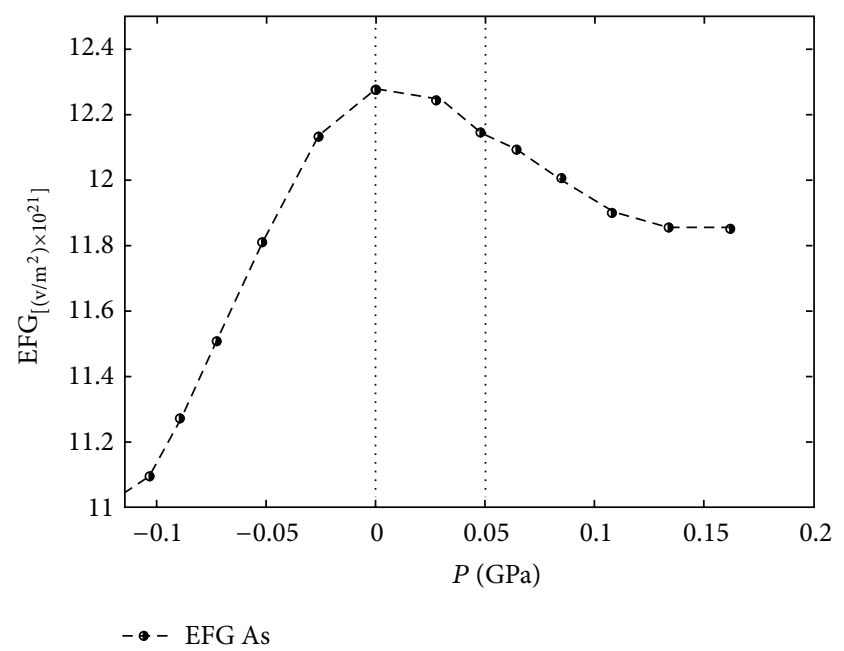

FIGURE 3: EFG values around the As nucleus at different pressures.

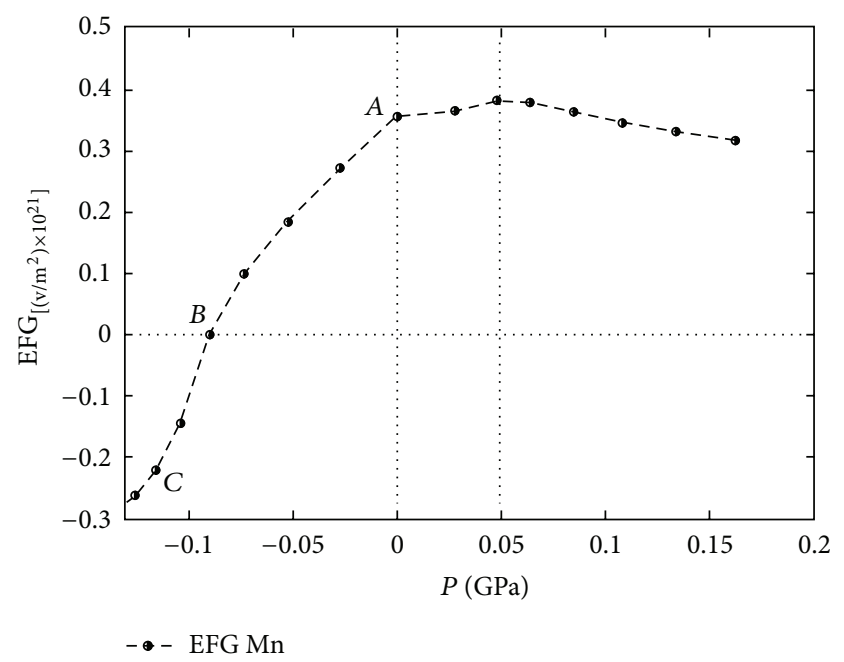

FIGURE 4: EFG values around the Mn nucleus at different pressures.

the $\mathrm{Mn}$ nucleus at $-0.0895 \mathrm{GPa}$ (the EFG is zero at this pressure) relative to 0 and $-0.115 \mathrm{GPa}$ is strongly modified. A comparison between the EFG values proves that slighting EFG at $-0.0895 \mathrm{GPa}$ corresponds to an electron charge density around the nucleus of Mn (Figure 5(b)) that is more symmetric.

On the other hand, by comparing Figures 5(a) and 5(c), the asymmetry of the electron charge density around the nucleus of $\mathrm{Mn}$ at $-0.115 \mathrm{GPa}$ is rotated by 45 degrees relative to each other. The negative value of the EFG at $-0.115 \mathrm{GPa}$ result from this rotation.

The study of contour plots in different planes provides useful information about the chemical bonding. In the following, some of this extracted information is noted. The contour plots around the Mn atoms in the (004) plane show that the electron charge density is placed mainly around special axes of the nucleus. This further determines that the bonding type of the Mn atoms is mostly covalent. However, the contour plot in this plane shows that at $-0.0895 \mathrm{GPa}$ 


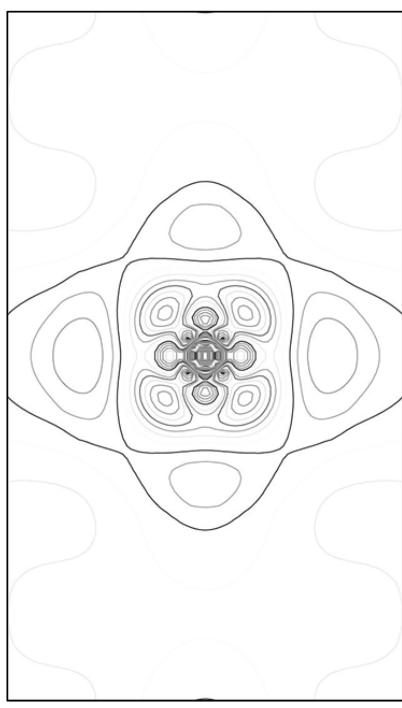

(a)

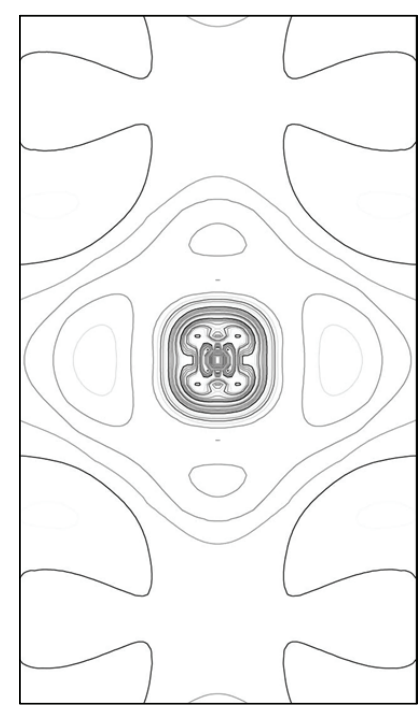

(b)

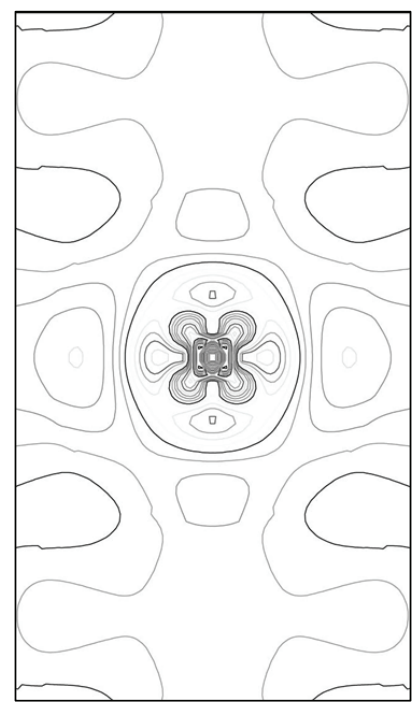

(c)

FIgURE 5: Contour plots of the different electric charge densities around the Mn atom in the (030) plane for three pressures.

the metallic bonding type around the $\mathrm{Mn}$ atom is increased. This corresponds to an increase of the crystal electron charge density symmetry. Therefore, the decreasing EFG value at $-0.0895 \mathrm{GPa}$ is completely understandable.

The covalent bonds have directional bonds with increased electron charge density along the bonds, whereas a metallic bonding is more uniform. The difference between As electronegativity (2.18) and Mn electronegativity (1.55) is less than 1.7 in Pauling electronegativity scale. Therefore, the bonding type of As-Mn is mostly covalent bonding. The source of electron charge density asymmetry at As nucleus is covalent bonds between As and Mn atoms. This is the main reason for the large EFG value at As nucleus. This asymmetry around As nucleus is obvious in the contour plots which contain As and Mn nuclear. Pressure affects the directional electron charge density asymmetry around the As nucleus. Then the EFG at the As nucleus subsequently decreases, but the EFG value dose not reach zero in the appropriate pressure range (Figure 3).

\section{The Effect of Pressure on HFF}

The $c / a$ ratio is kept constant in all calculations. It is predicted that the magnetic moment of the crystal at different pressures will be a constant value. The magnetic moment of the As atom is very small and almost constant, but, for the $\mathrm{Mn}$ atom and the total MnAs crystal, it decreases (Figure 6). For a study of this phenomenon, the HFF of Mn is calculated for the different pressures.

The calculated hyperfine fields arising from the Fermi contact interaction at zero fields are given in Table 1. These values agree with other values reported in [2]. In order to understand the origin of the hyperfine field, the site projected valence electron contribution as well as the core contributions are also given in this Table 1. Because of the opposite signs of the contributions from the core and valence electrons, the net

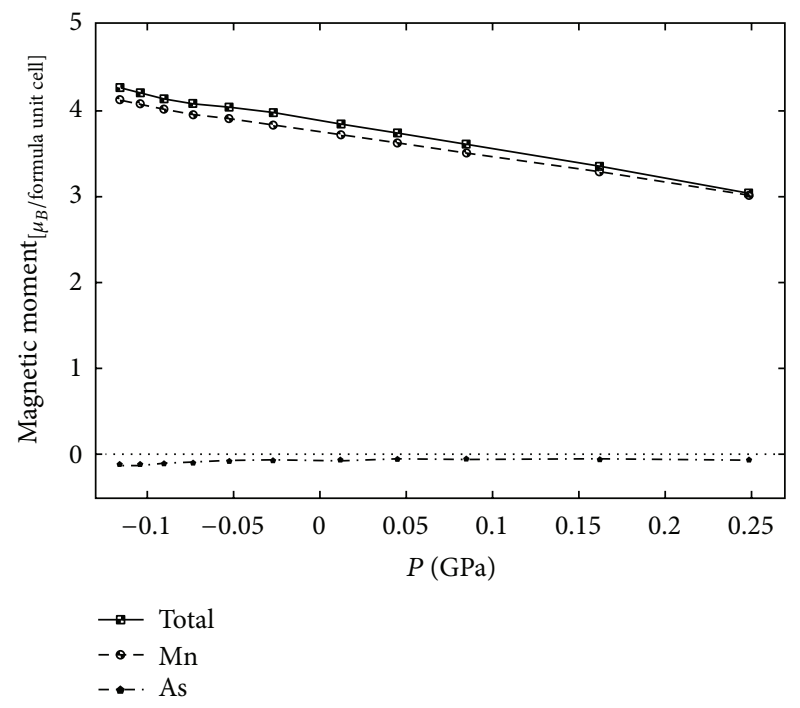

FIgURE 6: Magnetic moment of MnAs in the NiAs-type structure at different pressures.

TABLE 1: Calculated valence, core, and total contribution to the Fermi contact hyperfine field in kGauss at the Mn and As site.

\begin{tabular}{lccc}
\hline & Valence & Core & Total \\
\hline Mn & 581 & -453 & 128 \\
Reference [2] & 569 & -432 & 137 \\
As & 558 & 11 & 569 \\
Reference [2] & 563 & 5 & 568
\end{tabular}

field at the Mn site is much lower than the net field at the As site.

By increasing pressure, the HFF systematically increases. This systematic increment of HFF at the nucleus sites can be 


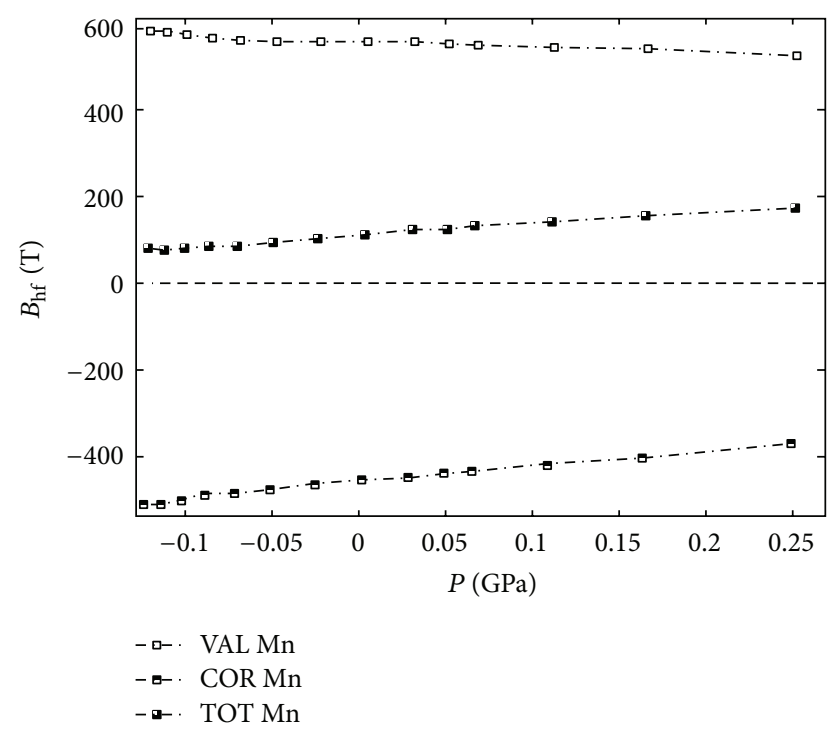

Figure 7: HFF values around the Mn nucleus at different pressures.

explained by the increased spin moment at the nucleus sites. When the pressure increases, the HFF at the nuclear sites increases almost linearly as shown in Figure 7.

The magnetic moment of the As nucleus is almost constant, but, for Mn nucleus and total MnAs, it decreases. The pressure localized valence electrons, increase electrons condense around the $\mathrm{Mn}$ nucleus and finally pinning the valance electrons. Therefore, the valance contribution of HFF decreases and then the magnetic moment of valence electrons decreases too (Figures 6 and 7).

The increase of electrons in the shell (core electrons) decreases the deference of spin-up and spin-down in the nucleus site because the electrons following the Pauli genuine, therefore the core contribution of HFF decreases to (2). The variations of core contribution are more than valance contribution, and total value of HFF is the sum of the valance and core contribution. Therefore, when pressure increases, the total HFF increases too (Figure 7).

\section{Conclusion}

The NiAs type has a stable structure of MnAs crystal at all thermodynamically accessible pressures. In order to prevent phase transition, the appropriate pressure range is $-0.126 \sim$ $0.4 \mathrm{GPa}$. The behavior of EFG depends on pressure variations. The asymmetry of the electron charge density distribution around the $\mathrm{Mn}$ and As nucleus varies with pressure. So EFG values vary with a transient slope. The range of these variations affects error correction of $Q$ measurement.

The research studies about magnetic moment behavior show that MnAs crystal magnetic properties not only depend on $c / a$ ratio but also depend on the distribution of electron charge density around its nucleus. It is possible to analyze magnetic moment variation based on pressure by the study of HFF behavior at the site nucleus.

\section{References}

[1] S. Sanvito and N. A. Hill, "Ground state of half-metallic zincblende MnAs," Physical Review B, vol. 62, no. 23, pp. 1555315560, 2000.

[2] P. Ravinran, A. Delin, P. James, and B. Johansson, "Magnetic, optical, and magneto-optical properties of $\mathrm{MnX}(\mathrm{X}=\mathrm{As}, \mathrm{Sb}$, or Bi) from full-potential calculations," Physical Review B, vol. 59, no. 24, Article ID 15680, 1999.

[3] A. Contineza, S. Picozzi, W. T. Geng, and A. J. Freeman, "Coordination and chemical effects on the structural, electronic, and magnetic properties in Mn pnictides," Physical Review B, vol. 64, Article ID 085204, 2001.

[4] M. Divis, K. Schwarz, and P. Blaha, "Rare earth borocarbides: Electronic structure calculations and electric field gradients," Physical Review B, vol. 62, no. 10, pp. 6774-6785, 2000.

[5] K. Maki, T. Kaneko, H. Hiroyoshi, and K. Kamigaki, "Crystalline and magnetic properties of MnAs under pressure," Journal of Magnetism and Magnetic Materials, vol. 177-181, no. 2, pp. 1361-1362, 1998.

[6] K. Schwarz, P. Blaha, and S. B. Trickey, "Electronic structure of solids with WIEN2k," Molecular Physics, vol. 108, no. 21-23, pp. 3147-3166, 2010.

[7] H. Haas and H. M. Petrilli, Physical Review B, vol. 61, no. 13, p. $588,2000$.

[8] G. J. Hill, J. M. Keartland, M. J. R. Hoch, and H. Haas, "Pressure dependence of the electric-field gradient in semimetallic arsenic and antimony: NQR measurements and full-potential linearized augmented-plane-wave calculations," Physical Review B, vol. 58, no. 20, pp. 13614-13618, 1998.

[9] S. Blugel, H. Akai, R. Zeller, and P. H. Dederichs, "Generalizedgradient-approximation description of band splittings in transition-metal oxides and fluorides," Physical Review B, vol. 49, pp. 10170-10175, 1994.

[10] P. Blaha, D. J. Singh, P. I. Sorantin, and K. Schwarz, "Electricfield-gradient calculations for systems with large extendedcore-state contributions," Physical Review B, vol. 46, no. 3, pp. 1321-1325, 1992.

[11] P. Blaha, K. Schwarz, G. K. H. Madson, D. Kvasnicka, and J. Luitz, WIEN2k, an Augmented Plane Wave Plus Local Orbitals Program for Calculating Crystal Properties, Vienna University of Technology, Vienna, Austria, 2001.

[12] H. Ohno, "Properties of ferromagnetic III-V semiconductors," Journal of Magnetism and Magnetic Materials, vol. 200, pp. 110119, 1999.

[13] T. Hayashi, Y. Hashimoto, S. Katsumuoto, and Y. Iye, "Effect of low-temperature annealing on transport and magnetism of diluted magnetic semiconductor (Ga,Mn)As," Applied Physics Letters, vol. 78, p. 1691, 2001.

[14] S. J. Potashinik, K. C. Ku, S. H. Chun, J. J. Berry, N. Samarth, and P. Schiffer, "Effects of annealing time on defect-controlled ferromagnetism in $\mathrm{Ga}_{1-x} \mathrm{Mn}_{x}$ As," Applied Physics Letters, vol. 79, p. 1495, 2001.

[15] G. Schmidt, D. Ferrand, L. W. Molenkamp, A. T. Filip, and B. J. Van Wees, "Fundamental obstacle for electrical spin injection from a ferromagnetic metal into a diffusive semiconductor," Physical Review B, vol. 62, no. 8, pp. R4790-R4793, 2000.

[16] K. Motizuki, K. Katoh, and A. Yanase, "Electronic band structures of NiAs-type compounds-I. Nonmagnetic state," Journal of Physics C, vol. 19, p. 495, 1986. 
[17] M. Shirai, T. Ogawa, I. Kitagawa, and N. Suzuki, "Band structures of zinc-blende-type MnAs and (MnAs) $)_{1}(\mathrm{GaAs})_{1}$ superlattice," Journal of Magnetism and Magnetic Materials, vol. 177-181, pp. 1383-1384, 1998.

[18] N. Menyuk, J. A. Kafalas, K. Dwight, and J. B. Goodenough, "Effects of pressure on the magnetic properties of MnAs," Physical Review, vol. 177, no. 2, pp. 942-951, 1969. 

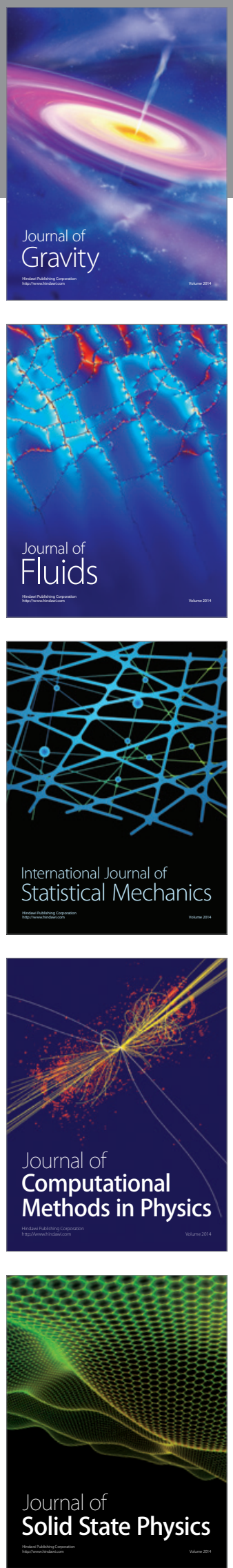

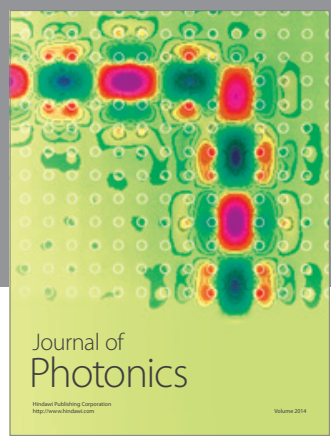

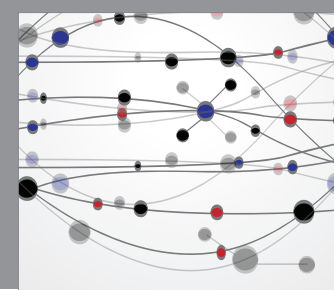

The Scientific World Journal

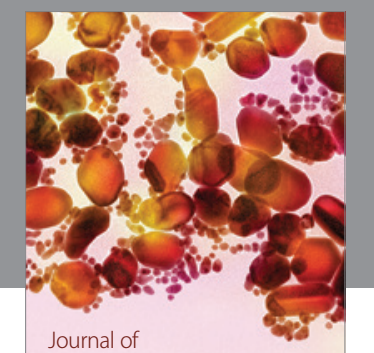

Soft Matter
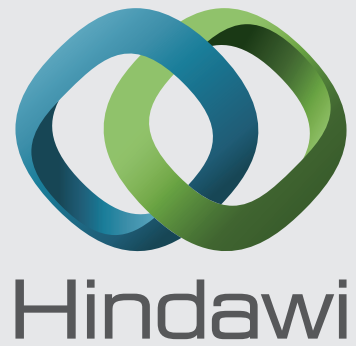

Submit your manuscripts at

http://www.hindawi.com
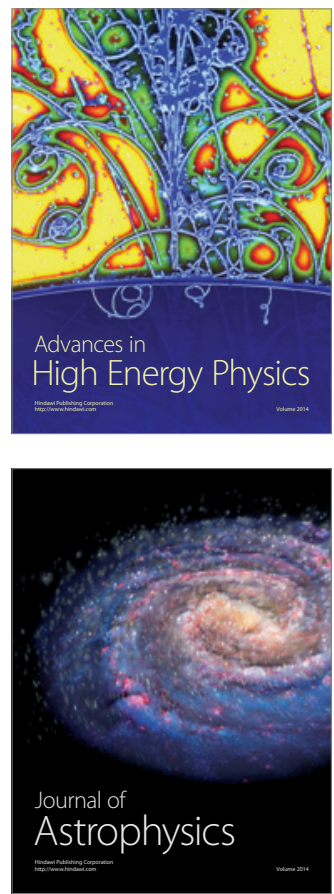
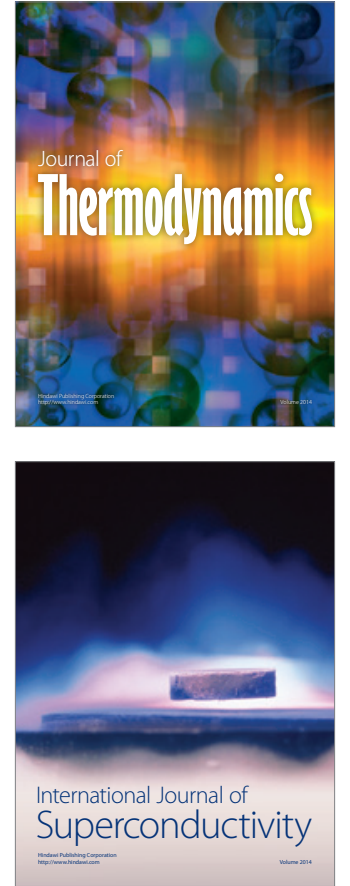
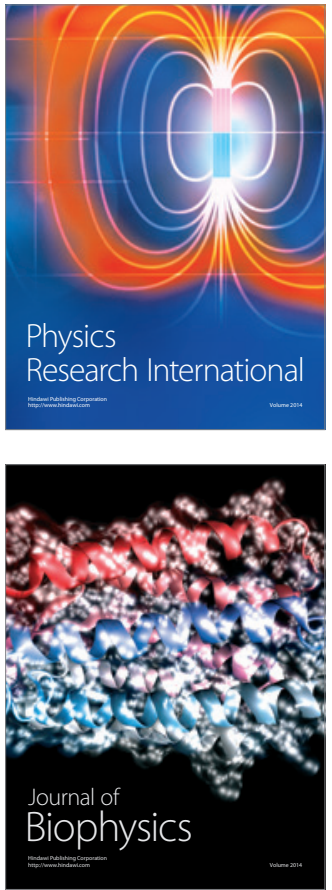
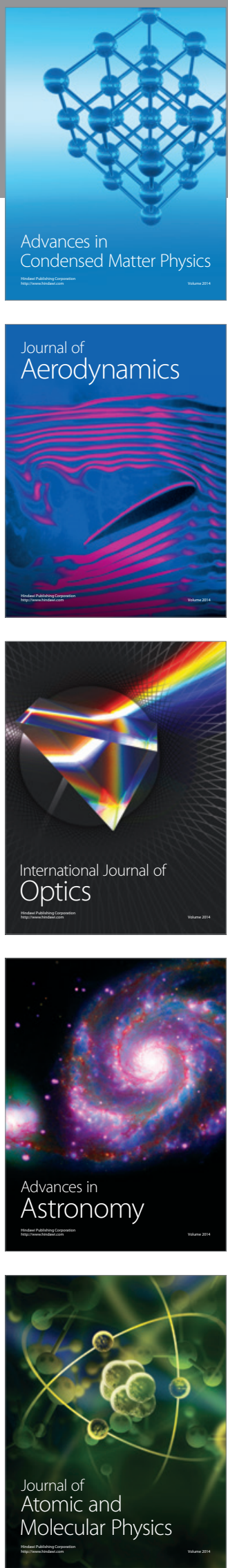